\title{
Fuzzy Upper Bounds in Groupoids
}

\author{
Sun Shin Ahn, ${ }^{1}$ Young Hee Kim, ${ }^{2}$ and J. Neggers ${ }^{3}$ \\ ${ }^{1}$ Department of Mathematics Education, Dongguk University, Seoul 100-715, Republic of Korea \\ ${ }^{2}$ Department of Mathematics, Chungbuk National University, Cheongju 361-763, Republic of Korea \\ ${ }^{3}$ Department of Mathematics, University of Alabama, Tuscaloosa, AL 35487-0350, USA
}

Correspondence should be addressed to Young Hee Kim; yhkim@chungbuk.ac.kr

Received 14 March 2014; Accepted 14 May 2014; Published 28 May 2014

Academic Editor: Young B. Jun

Copyright (C) 2014 Sun Shin Ahn et al. This is an open access article distributed under the Creative Commons Attribution License, which permits unrestricted use, distribution, and reproduction in any medium, provided the original work is properly cited.

\begin{abstract}
The notion of a fuzzy upper bound over a groupoid is introduced and some properties of it are investigated. We also define the notions of an either-or subset of a groupoid and a strong either-or subset of a groupoid and study some of their related properties. In particular, we consider fuzzy upper bounds in $\operatorname{Bin}(X)$, where $\operatorname{Bin}(X)$ is the collection of all groupoids. Finally, we define a fuzzy$d$-subset of a groupoid and investigate some of its properties.
\end{abstract}

\section{Introduction}

Iséki and Tanaka introduced two classes of abstract algebras: BCK-algebras and BCI-algebras [1,2].

Neggers and Kim [3] introduced the notion of $d$-algebras which is a useful generalization of BCK-algebras and then investigated several relations between $d$-algebras and BCKalgebras as well as several other relations between $d$-algebras and oriented digraphs. Han et al. [4] defined a variety of special $d$-algebras, such as strong $d$-algebras, (weakly) selective $d$-algebras, and others. The main assertion is that the squared algebra $(X ; \square, 0)$ of a $d$-algebra is a $d$-algebra if and only if the root $(X ; *, 0)$ of the squared algebra $(X ; \square, 0)$ is a strong $d$-algebra. Recently, Kim et al. [5] explored properties of the set of $d$-units of a $d$-algebra. It was noted that many $d$-algebras are weakly associative, and the existence of nonweakly associative $d$ /BCK-algebras was demonstrated. Moreover, we discussed the notions of a $d$-integral domain and a left-injectivity in $d / \mathrm{BCK}$-algebras.

The notion of the semigroup $(\operatorname{Bin}(X), \square)$ was introduced by Kim and Neggers [6]. Fayoumi [7] introduced the notion of the center $Z \operatorname{Bin}(X)$ in the semigroup $\operatorname{Bin}(X)$ of all binary systems on a set $X$ and showed that if $(X, \bullet) \in Z \operatorname{Bin}(X)$, then $x \neq y$ implies $\{x, y\}=\{x \cdot y, y \cdot x\}$. Moreover, she showed that a groupoid $(X, \bullet) \in Z \operatorname{Bin}(X)$ if and only if it is a locally zero groupoid. Han et al. [8] introduced the notion of hypergroupoids $(H \operatorname{Bin}(X), \square)$ and showed that $(H \operatorname{Bin}(X), \square)$ is a supersemigroup of the semigroup $(\operatorname{Bin}(X), \square)$ via the identification $x \leftrightarrow\{x\}$. They proved that $\left(\operatorname{HBin}^{*}(X), \ominus,[\emptyset]\right)$ is a $\mathrm{BCK}$-algebra.

\section{Preliminaries}

A d-algebra [3] is a nonempty set $X$ with a constant 0 and a binary operation "*" satisfying the following axioms:

(D1) $x * x=0$,

(D2) $0 * x=0$,

(D3) $x * y=0$ and $y * x=0$ imply $x=y$ for all $x, y \in X$.

A BCK-algebra is a $d$-algebra $X$ satisfying the following additional axioms:

(D4) $((x * y) *(x * z)) *(z * y)=0$,

(D5) $(x *(x * y)) * y=0$ for all $x, y, z \in X$.

Example 1 (see [4]). Consider the real numbers $\mathbf{R}$, and suppose that $(\mathbf{R} ; *, \mathbf{e})$ has the multiplication

$$
x * y=(x-y)(x-e)+e .
$$

Then $x * x=e ; e * x=e ; x * y=y * x=e$ yield $(x-y)(x-e)=$ $0,(y-x)(y-e)=0$ and $x=y$, or $x=e=y$; that is, $x=y$; that is, $(\mathbf{R} ; *, e)$ is a $d$-algebra. 
Given a nonempty set $X$, we let $\operatorname{Bin}(X)$ the collection of all groupoids $(X, *)$, where $*: X \times X \rightarrow X$ is a map and where $*(x, y)$ is written in the usual product form. Given elements $(X, *)$ and $(X, \bullet)$ of $\operatorname{Bin}(X)$, define a product “ $\square$ " on these groupoids as follows:

$$
(X, *) \square(X, \bullet)=(X, \square)
$$

where

$$
x \square y=(x * y) \cdot(y * x)
$$

for any $x, y \in X$. Using the notion, Kim and Neggers [6] showed the following theorem.

Theorem 2 (see [6]). (Bin $(X), \square)$ is a semigroup; that is, the operation " $\square$ " as defined in general is associative. Furthermore, the left-zero semigroup is an identity for this operation.

\section{Four Different Types of Fuzzy Subsets}

In what follows, let $X$ denote a groupoid unless otherwise specified.

Definition 3. Let $(X, *, 0)$ be a groupoid. A map $\mu$ of $X$ is called a fuzzy upper bound (or shortly, a fub) over $(X, *, 0)$ if $\mu(0) \geq \mu(x)$ for all $x \in X$. A map $\mu$ of $X$ is called a fuzzy lower bound (or shortly, a flb) over $(X, *, 0)$ if $\mu(0) \leq \mu(x)$ for all $x \in X$.

Example 4. Let $(X, *)$ be any groupoid and let $0 \in X$ such that $\mu(0)$ is the greatest element of $\mu(X):=\{\mu(x) \mid x \in X\}$ where $\mu: X \rightarrow[0,1]$. Then $\mu(0) \geq \mu(x)$ for all $x \in X$; that is, $\mu$ is a fub over $(X, *, 0)$.

Given a fub $\mu: X \rightarrow[0,1]$ over $(X, *, 0)$, we consider the following conditions: for any $x, y \in X$,

(A) $\mu(x * y)=\mu(y * x)=\mu(0)$ implies $\mu(x)=\mu(y)$,

(B) $\mu(x * y)=\mu(y * x)=\mu(0)$ implies $x=y$,

(C) $\mu(x * y)=\mu(y * x)$ implies $\mu(x)=\mu(y)$,

(D) $\mu(x * y)=\mu(y * x)$ implies $x=y$.

We have a diagram of implications as follows:

$$
\begin{array}{ccc}
(B) & \Longrightarrow & (A) \\
\Uparrow & & \Uparrow \\
(D) & \Longrightarrow & (C)
\end{array} .
$$

Thus we will look for counter examples to $(A) \Rightarrow(B),(B) \Rightarrow$ $(D),(A) \Rightarrow(C)$, and $(C) \Rightarrow(D)$.

Example 5. (a) Let $(X, *, 0)$ be a groupoid with $|X| \geq 2$ and let $\mu: X \rightarrow[0,1]$ be a constant map; that is, $\mu(x)=\alpha$ for some $\alpha \in[0,1]$ for all $x \in X$. Then $(A)$ holds as does $(C)$. On the other hand, $x \neq y$ implies $(B)$ fails as does $(D)$. Thus we have countered examples to $(A) \Rightarrow(B)$ and $(C) \Rightarrow(D)$. (b) Let $X:=\{0,1,2,3\}$ be a $d$-algebra with the following table:

\begin{tabular}{l|llll}
$*$ & 0 & 1 & 2 & 3 \\
\hline 0 & 0 & 0 & 0 & 0 \\
1 & 1 & 0 & 2 & 1 \\
2 & 2 & 2 & 0 & 3 \\
3 & 3 & 3 & 3 & 0
\end{tabular}.

Define a map $\mu: X \rightarrow[0,1]$ by

$$
\mu(x):= \begin{cases}1, & \text { if } x=0 \\ 0, & \text { if } x \neq 0 .\end{cases}
$$

It is easy to check that $\mu$ is a fub over $(X, *, 0)$ having the condition $(B)$. But $\mu$ does not have the condition $(D)$, since $\mu(1 * 2)=\mu(2)=\mu(2 * 1)$ and $1 \neq 2$. Hence $(B) \Rightarrow(D)$ is false.

(c) Let $X=2^{A}$, where $A=\{1,2,3,4\}$. Define a binary operation "*" on $X$ by $x * y:=\{t \mid t \in x, t \notin y\}, \forall x, y \in X$. Thus, if $x \subseteq y$, then $x * y=\emptyset$. Let $\mu(x):=1 /(1+|x|)$, where $|x|$ is the cardinality of $x$. Assume $\mu(x * y)=\mu(y * x)=\mu(\emptyset)$. Since $\mu(\emptyset)=1$, we have $|x * y|=0=|y * x|$ and so $x * y=$ $\emptyset=y * x$. Therefore $x \subseteq y, y \subseteq x$. Hence $x=y$. On the other hand, if $x=\{1,2,3\}, y=\{3,4,5\}$, then $x * y=\{1,2\}$, $y * x=\{4,5\}$, and $\mu(x * y)=1 / 3=\mu(y * x)$, but $x \neq y$. This shows that $(B)$ is true, but $(D)$ is false.

(d) Let $(X, *, 0)$ be a left-zero-semigroup; that is, for any $x, y \in X, x * y=x$, and let $\mu: X \rightarrow[0,1]$ be a fub over $(X, *, 0)$. Assume that $\mu(x * y)=\mu(y * x)$ for any $x, y \in X$. Since $(X, *, 0)$ is a left-zero-semigroup, we have $\mu(x)=\mu(x *$ $y)=\mu(y * x)=\mu(y)$. Hence condition $(C)$ holds for any fub $\mu$ over $(X, *, 0)$.

(e) Let $(X, *, 0)=(\mathbb{R},-, 0)$, where $\mathbb{R}$ is the set of all real numbers and "-" is the usual subtraction on $\mathbb{R}$. Also, if $\mu(x)=$ $e^{-x^{2}}$, then $\mu(0)=1 \geq \mu(x)$ for all $x \in \mathbb{R}$; that is, $\mu$ is a fub over $(\mathbb{R},-, 0)$. Note that $\mu(x-y)=\mu(y-x)=\mu(0)$ implies $x-y=y-x=0$ and $x=y$, so that $\mu(x)=\mu(y)$ as well; that is, the conditions $(B)$ and $(A)$ hold. Now if we let $y:=-1$ and $x:=2+y$, then $\mu(x-y)=\mu(2+y-y)=\mu(2)=$ $e^{-4}$ and $\mu(y-x)=\mu(y-2-y)=\mu(-2)=e^{-4}$, so that $\mu(x-y)=\mu(y-x)$. Since $e^{-4(y+1)}<1$ for any $y \neq-1$, we have $\mu(x)=\mu(2+y)=e^{-(2+y)^{2}}=e^{-y^{2}} e^{-4(y+1)}<e^{-y^{2}}=\mu(y)$. Thus $(C)$ fails to hold.

As a refinement of the condition $(A)$, we give two conditions $(A)^{*}$ and $(A)_{\alpha}^{*}, 0 \leq \alpha \leq 1$ as follows:

$$
\begin{aligned}
& (A)^{*} \mu(x * y)=\mu(y * x)=\mu(0) \text { implies } \mu(x)=\mu(y)=\mu(0), \\
& (A)_{\alpha}^{*} \mu(x * y)=\mu(y * x)=\mu(0) \text { implies } \mu(x)=\mu(y) \geq \alpha .
\end{aligned}
$$

Example 6. (a) If $\mu: X \rightarrow[0,1]$ is the constant map, that is, $\mu(x)=\alpha$ for all $x \in X$, then for any groupoid $(X, *, 0) \mu$ satisfies the condition $(A)^{*}$. Since $(A)^{*} \Rightarrow(A), \mu$ satisfies the condition $(A)$.

(b) Let $(X, *, 0):=(\mathbb{R},-, 0)$, where $\mathbb{R}$ is the set of all real numbers and "-" is the usual subtraction on $\mathbb{R}$, and let $\mu(x):=e^{-x^{2}}$. Then $\mu(0)=1 \geq \mu(x)>0$ for any $x \in \mathbb{R}$, and $\mu(x)=1$ if and only if $x=0$. Assume that $\mu(x-y)=$ $\mu(y-x)=\mu(0)$. Then $e^{-(x-y)^{2}}=e^{-(y-x)^{2}}=1$ and so $x-y=0$. 
Hence $x=y$. Therefore $\mu(x)=\mu(y)$; that is, $(A)$ holds. Let $x=y \neq 0$ imply $\mu(y)=\mu(x)=e^{-x^{2}}<1=\mu(0)$. Thus $(A)^{*}$ does not hold. Since $\lim _{x \rightarrow \infty} \mu(x)=0$, given $\alpha>0$, there is an $x$ such that $\mu(x)<\alpha$; that is, $(A)_{\alpha}^{*}$ does not hold for $\alpha>0$.

(c) For the groupoid $(\mathbb{R},-, 0)$, let $\mu_{\beta}(x):=\left(e^{-x^{2}}+\beta\right) /(1+$ $\beta$ ), where $\beta \geq 0$. Then $\mu_{\beta}(0)=1 \geq \mu_{\beta}(x)$ for all $x \in \mathbb{R}$ and $\lim _{x \rightarrow \infty} \mu_{\beta}(x)=\beta /(1+\beta) \leq \mu_{\beta}(x)$ for all $x \in X$. If we take $\alpha:=\beta /(1+\beta)$, then $\mu_{\beta}(x) \geq \alpha$ for all $x \in X$. If we assume $\mu_{\beta}(x-y)=\mu_{\beta}(y-x)=\mu_{\beta}(0)$, then $(x-y)^{2}=(y-x)^{2}=0$ and hence $\mu_{\beta}(x)=\mu_{\beta}(y)$; that is, the condition $(A)_{\alpha}^{*}$ holds. If $\alpha_{1}<$ $\alpha_{2}$, then $(A)_{\alpha_{2}}^{*} \Rightarrow(A)_{\alpha_{1}}^{*}$ holds clearly. If we set $\beta_{1}:=\alpha_{1} /\left(1-\alpha_{1}\right)$ and $\beta_{2}:=\alpha_{2} /\left(1-\alpha_{2}\right)$, then $\beta_{1}<\beta_{2}$ and $\lim _{x \rightarrow \infty} \mu_{\beta_{1}}(x)=$ $\lim _{x \rightarrow \infty}\left(\left(e^{-x^{2}}+\beta_{1}\right) /\left(1+\beta_{1}\right)\right)=\left(\beta_{1}\right) /\left(1+\beta_{1}\right)=\alpha<\alpha_{2}$. Take an $x \in \mathbb{R}$ so that $\alpha_{1}<\mu_{\beta_{1}}(x)<\alpha_{2}$. Then $e^{-x^{2}}+\beta_{1}<$ $\alpha_{2}\left(1+\beta_{1}\right)$. It follows that $e^{-x^{2}}<\left(\beta_{2} /\left(1+\beta_{2}\right)\right)\left(1+\beta_{1}\right)-\beta_{1}<$ $\left(\beta_{2}\left(1+\beta_{1}\right)-\beta_{1}\left(1+\beta_{2}\right)\right) /\left(1+\beta_{2}\right)=\left(\beta_{2}-\beta_{1}\right)\left(1+\beta_{1}\right) /(1+$ $\left.\beta_{2}\right)$; that is, $x^{2}>\ln \left[\left(1+\beta_{2}\right) /\left(\beta_{2}-\beta_{1}\right)\left(1+\beta_{1}\right)\right]$. Hence, if $|x|>\sqrt{\ln \left[\left(1+\beta_{2}\right) /\left(\beta_{2}-\beta_{1}\right)\left(1+\beta_{1}\right)\right]}$, then $(A)_{\alpha_{1}}^{*} \Rightarrow(A)_{\alpha_{2}}^{*}$ does not hold.

Theorem 7. Suppose that $\mu_{1}$ and $\mu_{2}$ are fubs over a groupoid $(X, *, 0)$ and that they both satisfy the condition $(A)$. If $\mu:=$ $\lambda \mu_{1}+(1-\lambda) \mu_{2}$, where $0 \leq \lambda \leq 1$, then $\mu$ is also a fub over $(X, *, 0)$ having the condition $(A)$.

Proof. Let $0<\lambda<1$. For any $x \in X$, we have $\mu(0)=\lambda \mu_{1}(0)+$ $(1-\lambda) \mu_{2}(0) \geq \lambda \mu_{1}(x)+(1-\lambda) \mu_{2}(x)=\mu(x)$. Hence $\mu$ is a fub over $(X, *, 0)$.

Assume that $\mu(x * y)=\mu(y * x)=\mu(0)$, but $\mu_{1}(x * y)<$ $\mu_{1}(0)$ or $\mu_{2}(x * y)<\mu_{2}(0)$. If $\mu_{1}(x * y)<\mu_{1}(0)$, then $\lambda \mu_{1}(x *$ $y)<\lambda \mu_{1}(0)$ and thus $(1-\lambda) \mu_{2}(x * y)=\mu(x * y)-\lambda \mu_{1}(x * y)>$ $\mu(x * y)-\lambda \mu_{1}(0)=\mu(0)-\lambda \mu_{1}(0)=(1-\lambda) \mu_{2}(0)$. This shows that $\mu_{2}(x * y)>\mu_{2}(0)$, which is a contradiction. Similarly, $\mu_{2}(x * y)>\mu_{2}(0)$ implies $\mu_{1}(x * y)>\mu_{1}(0)$, which is also a contradiction. Hence we obtain $\mu_{1}(x * y)=\mu_{1}(0), \mu_{2}(x * y)=$ $\mu_{2}(0)$. Similarly, we obtain $\mu_{1}(y * x)=\mu_{1}(0), \mu_{2}(y * x)=\mu_{2}(0)$. Since $\mu_{i}$ has the condition $(A)$, we have $\mu_{i}(x)=\mu_{i}(y)(i=$ $1,2)$. Hence $\mu(x)=\lambda \mu_{1}(x)+(1-\lambda) \mu_{2}(x)=\lambda \mu_{1}(y)+(1-$ ג) $\mu_{2}(y)=\mu(y)$. Thus $\mu$ has the condition $(A)$.

Theorem 8. Suppose that $\mu_{1}$ and $\mu_{2}$ are fubs over a groupoid $(X, *, 0)$ and that $\mu_{2}$ has the condition $(A)^{*}$ with $\mu_{1}(0)<\mu_{2}(0)$. If $\mu:=\mu_{1} \vee \mu_{2}$, where $\left(\mu_{1} \vee \mu_{2}\right)(x):=\max \left\{\mu_{1}(x), \mu_{2}(x)\right\}$, then $\mu$ is a fub over $(X, *, 0)$ having the condition $(A)^{*}$

Proof. If $\mu=\mu_{1} \vee \mu_{2}$, then $\mu(0)=\mu_{1}(0) \vee \mu_{2}(0), \mu(x)=$ $\mu_{1}(x) \vee \mu_{2}(x)$. If $\mu_{1}(x) \geq \mu_{2}(x)$, then $\mu(x)=\mu_{1}(x)$ and $\mu(0) \geq$ $\mu_{1}(0) \geq \mu_{1}(x)=\mu(x)$ implies $\mu(0) \geq \mu(x)$ for any $x \in X$. Similarly, if $\mu_{2}(x) \geq \mu_{1}(x)$, then $\mu(0) \geq \mu(x)$ for any $x \in X$. Hence $\mu$ is a fub over $(X, *, 0)$

Assume that $\mu(x * y)=\mu(y * x)=\mu(0)$. Then $\mu_{1}(x *$ $y) \vee \mu_{2}(x * y)=\mu(x * y)=\mu(0)=\mu_{1}(0) \vee \mu_{2}(0)=\mu_{2}(0)$. If $\mu_{2}(x * y)<\mu_{2}(0)$, then $\mu_{1}(x * y)=\mu_{2}(0)$ and $\mu_{1}(0) \geq \mu_{1}(x *$ $y)=\mu_{2}(0)$, which is a contradiction. Hence $\mu_{2}(x * y) \geq \mu_{2}(0)$; that is, $\mu_{2}(x * y)=\mu_{2}(0)$. Similarly, $\mu(y * x)=\mu(0)$ implies $\mu_{2}(0)=\mu_{2}(y * x)$. Hence $\mu_{2}(x * y)=\mu_{2}(y * x)=\mu_{2}(0)$. Since $\mu_{2}$ has the condition $(A)^{*}$, we have $\mu_{2}(x)=\mu_{2}(y)=\mu_{2}(0)$ and so $\mu(x)=\mu(0)$. Similarly, we obtain $\mu(y)=\mu(0)$. Thus $\mu$ has the condition $(A)^{*}$.

Example 9. (a) Suppose $A$ and $B$ are subsets of $X$ such that $A \backslash B, A \cap B$, and $B \backslash A$ are nonempty. Let $0 \in A \cap B$ and let $\mu_{1}:=\chi_{A}, \mu_{2}:=\chi_{B}$, where $x \in A \backslash B, y \in B \backslash A$, and $(X, *)$ is the left-zero-semigroup. It follows that $\mu(x * y)=$ $\mu_{1}(x * y) \vee \mu_{2}(x * y)=\mu(x)=\mu_{1}(x) \vee \mu_{2}(x)=1 \vee 0=1$ and that $\mu(y * x)=1=\mu(y), \mu(0)=1=\mu_{1}(1)=\mu_{2}(1)$. Hence $\mu(x)=\mu(y)=\mu(0)$; that is, the condition $(A)^{*}$ holds.

(b) Let $(\mathbb{R},-, 0)$ be the set of all real numbers equipped with subtraction and the zero element 0 . Let $\mu_{1}(x):=1+x-$ $\lceil x\rceil$ and $\mu_{2}(x):=1+\lfloor x\rfloor-x$. If $x \in \mathbb{Z}$, then $x=\lceil x\rceil=\lfloor x\rfloor$ and hence $\mu_{1}(x)=\mu_{2}(x)=1=\mu(0)$. If $x=n+\alpha$ where $n \in \mathbb{Z}, 0<\alpha<1 / 2$, then $\mu_{1}(x)=\alpha$ and $\mu_{2}(x)=1-\alpha$. Hence $\mu(x)=\mu_{1}(x) \vee \mu_{2}(x)=1-\alpha$. If $x=n+\beta$ where $n \in \mathbb{Z}, 1 / 2<\beta<1$, then $\mu_{1}(x)=\beta$ and $\mu_{2}(x)=1-\beta$. Hence $\mu(x)=\mu_{1}(x) \vee \mu_{2}(x)=\beta$. Assume $\mu(x-y)=\mu(y-x)=\mu(0)$. Since $\mu(0)=1$, we have $x-y, y-x \in \mathbb{Z}$, say $y-x=n \in \mathbb{Z}$. If we let $x:=m+\gamma$ where $m \in \mathbb{Z}, 0<\gamma<1$, then $y=x+n=$ $(n+m)+\gamma$. It follows that $\mu(x)=\mu(y)$ has the value either $\gamma$ or $1-\gamma$. Hence $\mu$ satisfies the condition $(A)$, but not the condition $(A)^{*}$.

Note that, in Example 9 (b), we know that $\mu_{1}(0)=\mu_{2}(0)=$ 1. This shows that the condition $\mu_{1}(0)<\mu_{2}(0)$ is a very necessary condition in Theorem 8 .

\section{Either-or in Groupoids}

Definition 10. Let $(X, *)$ be a groupoid and let $\emptyset \neq U \subseteq X$. Then $U$ is said to be either-or if $x * y \in U, y * x \in U$ implies either $\{x, y\} \subseteq U$ or $\{x, y\} \subseteq U^{c}$ for any $x, y \in X$.

Proposition 11. Suppose that $\mu$ is a fub over a groupoid $(X, *, 0)$ and that $\mu$ satisfies the condition $(A)$. Let $\operatorname{Ker} \mu:=$ $\{x \in X \mid \mu(x)=\mu(0)\}$. Then Ker $\mu$ is an either-or subset of $X$.

Proof. Let $x * y, y * x \in \operatorname{Ker} \mu$ for any $x, y \in X$. Then $\mu(x * y)=$ $\mu(0)=\mu(y * x)$. Since $\mu$ satisfies the condition $(A)$, we have $\mu(x)=\mu(y)$. Thus, also, if $x \in \operatorname{Ker} \mu$, then $y \in \operatorname{Ker} \mu$, and if $x \notin \operatorname{Ker} \mu$, then $y \notin \operatorname{Ker} \mu$. Therefore $\operatorname{Ker} \mu$ is an either-or subset of $X$.

Note that $0 \in \operatorname{Ker} \mu$ in Proposition 11.

Definition 12. An either-or subset $U$ is said to be with alternative if there are elements $\{x, y\} \subseteq U,\{u, v\} \subseteq U^{c}$ such that $\{x * y, y * x\} \subseteq U,\{u * v, v * u\} \subseteq U$.

Example 13. If $(\mathbb{Z},+)$ is the groupoid of all integers with respect to the usual addition and if $U:=2 \mathbb{Z}$ is the set of all even integers, then $U$ is an either-or subset of $X$. In fact, let $x+y=y+x=2 u \in U$ and $x=2 v$, where $u, v \in \mathbb{Z}$. Then $y=2 u-2 v=2(u-v) \in U$. Thus, $U$ is an either-or subset of $(\mathbb{Z},+)$. On the other hand, $U^{c}$ consists of all odd integers. Now if $x+y=y+x$ is odd for any $x, y \in \mathbb{Z}$ and if $x$ is odd, then $y=(x+y)-x$ is even. If $x$ is even, then $y$ is odd. 
Hence $U^{c}$ fails to be an either-or subset of $(\mathbb{Z},+)$. The subset $U=2 \mathbb{Z}$ of $(\mathbb{Z},+)$ is an either-or subset with alternative. Both $\mathbb{Z}$ and $\mathbb{Z}^{c}=\emptyset$ are either-or subsets, but without alternative.

Proposition 14. Let $0 \in U$ be a nonempty subset of a groupoid $(X, *)$. Suppose that $U$ is an either-or subset of the groupoid $(X, *)$ and that $\mu=\chi_{U}$ is the characteristic function of $U$; that is, $\mu(x)=1$ if $x \in U$ and $\mu(x)=0$ otherwise. Then $\mu$ satisfies the condition $(A)$.

Proof. Let $0 \in U$ be a "selected" element; that is, $\mu(0)=1$. Then $\mu(x * y)=\mu(y * x)=\mu(0)$ implies $x * y, y * x \in U$. Since $U$ is an either-or subset of the groupoid $(X, *)$, we have either $\{x, y\} \subseteq U$ or $\{x, y\} \subseteq U^{c}$; that is, $\mu(x)=\mu(y)=1$ or $\mu(x)=\mu(y)=0$. In any case $\mu(x)=\mu(y)$ and the condition (A) holds.

Let $(X, *)$ be a groupoid and let $\emptyset \neq S \subseteq X$. Let $\mathrm{EO}(S)$ be the collection of all either-or subsets of the groupoid $(X, *)$, sometimes denoted as $\mathrm{EO}(S ;(X, *))$, which contain the subset $S$ of $X$. Then, since $(X, *)$ is an either-or subset of $(X, *)$, it follows that $\operatorname{EOR}(S):=\cap_{i}\left\{U_{i} \mid U_{i} \in \operatorname{EO}(S ;(X, *))\right\} \supseteq$ S.

Proposition 15. Let $(X, *)$ be a groupoid and let $\emptyset \neq S \subseteq U$. Then $\operatorname{EOR}(S ;(X, *))$ is an either-or subset of $(X, *)$ containing S.

Proof. Let $x, y \in X$ such that $x * y, y * x \in \operatorname{EOR}(S ;(X, *))$. If $\{x, y\} \subseteq U^{c}$ for some either-or subset $U$ of $(X, *)$ which contains $S$, then $\operatorname{EOR}(S ;(X, *)) \subseteq U$ implies $U^{c} \subseteq$ $\operatorname{EOR}(S,(X, *))^{c}$ and $\{x, y\} \subseteq \operatorname{EOR}(S,(X, *))^{c}$. If this is not the case, then $\{x, y\} \nsubseteq U^{c}$ for any either-or subset $U$ of $(X, *)$ containing $S$, whence $\{x, y\} \subseteq U$. Thus $\{x, y\} \subseteq$ $\operatorname{EOR}(S ;(X, *))$. This shows that $\operatorname{EOR}(S ;(X, *))$ is an eitheror subset of $(X, *)$.

We have the following corollaries.

Corollary 16. Let $(X, *)$ be a groupoid. If $\emptyset \neq S \subseteq T$, then $\operatorname{EOR}(S ;(X, *)) \subseteq \operatorname{EOR}(T ;(X, *))$.

Corollary 17. Let $(X, *)$ be a groupoid. Then we have

$$
\begin{gathered}
\emptyset=\operatorname{EOR}(\emptyset ;(X, *)) \subseteq \operatorname{EOR}(\{0\} ;(X, *)) \\
\subseteq \cdots \subseteq \operatorname{EOR}(X ;(X, *))=X .
\end{gathered}
$$

Example 18. (a) Let $(X, *)$ be the left-zero-semigroup; that is, $x * y=x$ for all $x, y \in X$, and let 0 be a fixed element for which $\mu(0) \geq \mu(x)$ for all $x \in X$, where $\mu: X \rightarrow[0,1]$ is a fuzzy subset of $X$. Then $\mu(x * y)=\mu(x)=\mu(y * x)=\mu(y)=\mu(0)$ implies $\mu(x)=\mu(y)=\mu(0)$ and $\mu$ satisfies the condition $(A)^{*}$. If $U$ is any nonempty subset of $X$, then $x * y, y * x \in U$ implies $\{x, y\} \subseteq U$, so that $U$ is an either-or subset without alternative. Hence $\operatorname{EOR}(U ;(X, *))=U$ for any $\emptyset \neq U \subseteq X$ whatever. Of course, if $(X, *, 0)$ is the right-zero-semigroup, that is, $x * y=y$ for any $x, y \in X$, then the same conclusions hold.

(b) Let $(\mathbb{Z},+, 0)$ be the group of all integers with respect to addition. We claim that $n \mathbb{Z}=\{n x \mid x \in \mathbb{Z}\}$ is an either-or subset of $(\mathbb{Z},+, 0)$. Given $n \in \mathbb{Z}$, if $n=0$, and if $x+y, y+x \in$ $\{0\}$ and $x=0$, then $y=0$ and hence $\{x, y\}=\{0\} \subseteq\{0\}$. On the other hand, if $x \neq 0$, then $y=-x$ implies $\{x, y\}=\{x,-x\} \subseteq$ $\{0\}^{c}=\mathbb{Z}-\{0\}$. Hence $\{0\}=0 \mathbb{Z}$ is an either-or subset of the groupoid $(\mathbb{Z},+, 0)$. Let $n \neq 0$. If $x+y=y+x=n u$, and if $x=n v$, then $y=(x+y)-x=n u-n v=n(u-v)$; that is, $y \in n \mathbb{Z}$ as well. Thus $\{x, y\} \subseteq n \mathbb{Z}$. Assume that $x \notin n \mathbb{Z}$; that is, $x=n u^{\prime}+\alpha$ for some $u^{\prime} \in \mathbb{Z}$ and $\alpha \in \mathbb{Z}$ such that $0<\alpha<n$. Then $y=n u-x=n\left(u-u^{\prime}\right)-\alpha \notin n \mathbb{Z}$. Hence $\{x, y\} \subseteq(n \mathbb{Z})^{c}$.

We claim that $\operatorname{EOR}(\{n\} ;(\mathbb{Z},+))=n \mathbb{Z}$ for all $n \in$ $\mathbb{Z}$. Since $\{0\}$ is the smallest either-or subset of $(\mathbb{Z},+)$, $\operatorname{EOR}(\{0\} ;(\mathbb{Z},+))=\{0\}=0 \mathbb{Z}$. Let $n \neq 0$ in $\mathbb{Z}$. Then $0 \in$ $\operatorname{EOR}(\{n\} ;(\mathbb{Z},+))$. In fact, if $U_{i}$ is an either-or subset of $(\mathbb{Z},+)$ containing $n$, then either $\{n, 0\} \subseteq U_{i}$ or $\{n, 0\} \subseteq U_{i}^{c}$, since $n+0=0+n=n \in U_{i}$. It follows that $0 \in U_{i}$ for all either-or subset $U_{i}$ of $(\mathbb{Z},+)$; that is, $0 \in \operatorname{EOR}(\{n\} ;(\mathbb{Z},+))$.

We claim that $-n \in \operatorname{EOR}(\{n\} ;(\mathbb{Z},+))$. If $U_{i}$ is an either-or subset of $(\mathbb{Z},+)$, either $\{n,-n\} \subseteq U_{i}$ or $\{n,-n\} \subseteq U_{i}^{c}$ for any either-or subset $U_{i}$ of $(\mathbb{Z},+)$, since $n+(-n)=(-n)+n=0 \in U_{i}$. Since $n \in U_{i}$, we have $-n \in U_{i}$ for all either-or subset $U_{i}$. This proves that $-n \in \operatorname{EOR}(\{n\} ;(\mathbb{Z},+))$. Hence $\{-n, 0, n\} \subseteq$ $\operatorname{EOR}(\{n\} ;(\mathbb{Z},+))$.

Assume that $\mathbb{B}$ is an either-or subset of $(\mathbb{Z},+)$ containing $n$. Then it is easy to see that $0,-n \in \mathbb{B}$. If we let $x:=n, y:=$ $-2 n$, then $x+y=y+x=-n \in \mathbb{B}$. Since $x=n \in \mathbb{B}$ and $\mathbb{B}$ is an either-or subset of $(\mathbb{Z},+)$, we obtain $-2 n=y \in \mathbb{B}$. Similarly, if we let $x=-n$ and $y:=2 n$, then we obtain $2 n=y \in \mathbb{B}$. Similarly, we obtain $\pm 3 n, \pm 4 n, \ldots, \in \mathbb{B}$. Thus $n \mathbb{Z} \subseteq \mathbb{B}$; that is, $\mathbb{B}$ is an either-or subset of $(\mathbb{Z},+)$ containing $n \mathbb{Z}$. It follows that $n \mathbb{Z}=\cap\left\{U_{i} \mid U_{i}\right.$ : either-or subset containing $\left.n\right\}=$ $\operatorname{EOR}(\{n\} ;(\mathbb{Z},+))$.

(c) Let $\mathbb{N}:=\{1,2,3, \ldots\}$ and let $a \in \mathbb{N}$. If $x+y=a$, then $x<a, y<a$ and thus $\{a\}$ is an either-or subset of $\mathbb{N}$ without alternative, since $\{x, y\} \subseteq\{a\}^{c}$. If $U_{a}^{k}:=\{a, 2 a, \ldots, k a\}$ for any $a \in \mathbb{N}$, let $x+y=y+x \in U_{a}^{k}$. Then there exists $h \in \mathbb{N}$ such that $x+y=h a, h \leq k$. If $x=j a, j<h$, then $y=h a-x=$ $(h-j) a \in U_{a}^{k}$. Hence $\{x, y\} \subseteq U_{a}^{k}$. If $x \notin U_{a}^{k}$, then $y \notin U_{a}^{k}$ and $\{x, y\} \subseteq\left(U_{a}^{k}\right)^{c}$, so that $U_{a}^{k}$ is an either-or subset of $\mathbb{N}$ with alternative if $k \geq 2$.

Proposition 19. Let $(X, *)$ be a groupoid and let $U \subseteq X$ such that $x * y, y * x \in U$ implies $x=y$. Then $U$ is an either-or subset of $(X, *)$.

Proof. Let $x * y, y * x \in U$ for any $x, y \in X$. By assumption, we have $x=y$ and so $\{x, y\}=\{x\}$. Hence either $\{x\} \subseteq U$ or $\{x\} \subseteq U^{c}$. Thus $U$ is an either-or subset of $X$. $(X, *)$.

We call such a subset $U$ of $X$ a strong either-or subset of

Example 20. (a) Let $(X, *, f)$ be a leftoid, and let $U=\{a\}$ be a singleton. If $x * y=f(x) \in U$ and $y * x=f(y) \in U$, then $f(y)=f(x)=a$. Hence, if $\left|f^{-1}(a)\right|=1$, then $x=y$, and $U$ is a strong either-or subset of $(X, *)$.

(b) Let $\mathbb{N}$ be the set of all natural numbers and "+" be the usual addition on $\mathbb{N}$. If $U:=\{2\}$, then $U$ is a strong either-or subset of $\mathbb{N}$. In fact, if $x+y=y+x \in U$, then $x+y=y+x=2$ and hence $x=y=1$. 
Proposition 21. Let $(X, *, 0)$ be a groupoid and let $U$ be a strong either-or subset of $X$ with $0 \in U$. If $\mu:=\chi_{U}$ is the characteristic function of $U$, then $\mu$ is a fub over $(X, *, 0)$. Furthermore, $\mu$ satisfies the condition $(B)$ and $\operatorname{Ker} \mu=U$.

Proof. Since $0 \in U$, we have $\mu(0)=1 \geq \mu(x)$ for all $x \in X$ and $\mu$ is a fub over $(X, *, 0)$. Let $\mu(x * y)=\mu(y * x)=\mu(0)$ for any $x, y \in X$. Since $\mu(0)=1$, we have $x * y, y * x \in$ $U$. Hence $x=y$, since $U$ is a strong either-or subset of $X$. Therefore $\mu$ satisfies the condition $(B)$ and in that case $\operatorname{Ker} \mu=$ $\mu^{-1}(\mu(0))=U$.

Proposition 22. Let $(X, *, e)$ be a group all of whose elements have finite order and let $\mu: X \rightarrow[0,1]$ be a fuzzy subgroup. Then $\mu$ has the condition $(A)$ and $\operatorname{Ker} \mu$ is an either-or subset of $X$.

Proof. Let $\mu$ be a fuzzy subgroup of $X$. Then $\mu(x * y) \geq$ $\min \{\mu(x), \mu(y)\}$ for any $x, y \in X$. Thus $\mu\left(x^{2}\right)=\mu(x * x) \geq$ $\mu(x)$ and $\mu\left(x^{n}\right) \geq \min \left\{\mu\left(x^{n-1}\right), \mu(x)\right\}$. By induction $\mu\left(x^{n-1}\right) \geq$ $\mu(x)$ implies $\mu\left(x^{n}\right) \geq \mu(x)$, so that $\mu(e)=\mu\left(x^{n}\right) \geq \mu(x)$ implies $\mu(e) \geq \mu(x)$ for all $x \in X$; that is, $\mu$ is a fub over $(X, *, e)$.

If $\operatorname{Ker} \mu=\mu^{-1}(\mu(e))=U$, then $\mu(x * y)=\mu(y * x)=\mu(e)$ implies $\mu\left((x * y) * y^{-1}\right)=\mu(x) \geq \min \left\{\mu(x * y), \mu\left(y^{-1}\right)\right)$ and hence $\mu(x) \geq \mu\left(y^{-1}\right) \geq \mu(y)$, so that $\mu(x)=\mu(y)$; the condition $(A)$ holds for $\mu$, and Ker $\mu$ is an either-or subset of $(X, *, e)$.

\section{Fuzzy Upper Bounds in $\operatorname{Bin}(X)$}

Theorem 23. Let $\mu$ be a fub over $(X, *, 0)$ with the condition $(A)$ and a fub over $(X, \bullet, 0)$ with the condition $(A)^{*} . \operatorname{If}(X, \square):=$ $(X, *) \square(X, \bullet)$, then $\mu$ is a fub over $(X, \square, 0)$ with the condition $(A)^{*}$.

Proof. Assume that $\mu(x \square y)=\mu(y \square x)=\mu(0)$ for any $x, y \in$ $X$. Then $\mu((x * y) \bullet(y * x))=\mu((y * x) \bullet(x * y))=\mu(0)$ implies $\mu(x * y)=\mu(y * x)=\mu(0)$, so that $\mu(x)=\mu(y)=\mu(0)$. Hence $\mu$ satisfies the condition $(A)^{*}$ over $(X, \square, 0)$.

Corollary 24. Let $\mu$ be a fub over both $(X, *, 0)$ and $(X, \bullet, 0)$ with the condition $(A)^{*}$. If $(X, \square):=(X, *) \square(X, \bullet)$, then $\mu$ is an lub over $(X, \square, 0)$ with the condition $(A)^{*}$.

Proof. Straightforward.

Let $X$ be a nonempty set. If $\langle\mu ;(A)\rangle$ and $\left\langle\mu ;(A)^{*}\right\rangle$ are the collections of groupoids $(X, *, 0)$ such that $\mu$ satisfies the condition $(A)$ (resp., $\left.(A)^{*}\right)$, then $\left\langle\mu ;(A)^{*}\right\rangle \subseteq\langle\mu ;(A)\rangle$ and

$$
\begin{gathered}
\langle\mu ;(A)\rangle \square\left\langle\mu ;(A)^{*}\right\rangle \subseteq\langle\mu ;(A)\rangle, \\
\left\langle\mu ;(A)^{*}\right\rangle \square\left\langle\mu ;(A)^{*}\right\rangle \subseteq\left\langle\mu ;(A)^{*}\right\rangle ;
\end{gathered}
$$

that is, $\left(\left\langle\mu ;(A)^{*}\right\rangle, \square\right)$ is a subsemigroup of $(\operatorname{Bin}(X), \square)$.

Theorem 25. Let $\mu$ be a fub over $(X, *, 0)$ with the condition $(B)$, and let $\mu$ be a fub over $(X, \bullet, 0)$ with the condition $(A)^{*}$. If
$(X, \square):=(X, *) \square(X, \bullet)$, then $\mu$ is a fub over $(X, \square, 0)$ with the condition $(B)$.

Proof. Assume that $\mu(x \square y)=\mu(y \square x)=\mu(0)$ for any $x, y \in$ $X$. Then $\mu((x * y) \bullet(y * x))=\mu((y * x) \bullet(x * y))=\mu(0)$. Since $\mu$ satisfies the condition $(A)^{*}$ over $(X, \bullet, 0)$, we have $\mu(x * y)=$ $\mu(y * x)=\mu(0)$. Since $\mu$ has the condition $(B)$ over $(X, *, 0)$, we obtain $x=y$, proving the theorem.

\section{Fuzzy- $d$-Subsets in Groupoids}

Given a groupoid $(X, *, 0)$, the following are interesting properties in fuzzy subgroupoids $\mu: X \rightarrow[0,1]$ :

(1) $\mu(x * x)=\mu(0)$ for all $x \in X$,

(2) $\mu(0 * x)=\mu(0)$ for all $x \in X$,

(3) if $\mu(x * y)=\mu(y * x)=\mu(0)$, then $\mu(x)=\mu(y)$ for all $x, y \in X$.

Definition 26. A fub $\mu$ over a groupoid $(X, *)$ is called a fuzzy-d-subset of the groupoid $(X, *, 0)$ if it satisfies conditions (1), (2), and (3).

Example 27. (a) Let $X:=\mathbb{R}$ be the set of all real numbers and let "+" be the usual addition on $\mathbb{R}$. Then every fuzzy- $d$ subset $\mu$ of $(X,+, 0)$ is constant. In fact, for all $x \in X$, we have $\mu(0)=\mu(x+x)=\mu(2 x)$. If we let $y:=2 x$, then $\mu(y)=\mu(0)$ for all $y \in X$; that is, $\mu$ is a constant function on $X$.

(b) Let $(X, *, 0)$ be a group with identity 0 . Let $\mu$ be a fuzzy subset of $X$ with (2); then $\mu(x)=\mu(0 * x)=\mu(0)$ for all $x \in X$, whence $\mu$ is a constant function. Therefore $\mu$ is a fuzzy$d$-subset of $(X, *, 0)$.

Proposition 28. Let $(X, *, 0)$ be a d-algebra. If $\mu=\chi_{\{0\}}$ is the characteristic function of $\{0\}$; that is, $\mu(0)=1$ and $\mu(x)=0$ otherwise, then $\mu$ is a fuzzy-d-subset of $X$ and $\operatorname{Ker} \mu=\{0\}$.

Proof. Let $x, y \in X$. Then $\mu(x * x)=\mu(0), \mu(0 * x)=\mu(0)$. If $\mu(x * y)=\mu(y * x)=\mu(0)$, then $x * y=y * x=0$. Since $(X, *, 0)$ is a $d$-algebra, we obtain $x=y$. Hence $\mu(x)=\mu(y)$. This shows that $\mu$ is a fuzzy- $d$-subset of $(X, *, 0)$ and $\operatorname{Ker} \mu=$ $\mu^{-1}(\mu(0))=\mu^{-1}(1)=\{0\}$.

Proposition 29. Let $S$ be an either-or subset of a groupoid $(X, *, 0)$ such that $\Delta(X, *):=\{x * x \mid x \in X\}$ and $\Delta_{0}(X, *):=$ $\{0 * x \mid x \in X\}$ are subsets of $S$. Let $\mu:=\chi_{S}$ be the characteristic function of $S$ and $0 \in S$. Then $\mu$ is a fuzzy-d-subset of $(X, *, 0)$.

Proof. Since $0 \in S$, we have $\mu(0)=1 \geq \mu(x)$ for all $x \in$ $X$. Since $\Delta(X, *), \Delta_{0}(X, *) \subseteq S$, we obtain $\mu(x * x)=\mu(0)$, $\mu(0 * x)=\mu(0)$. Assume that $\mu(x * y)=\mu(y * x)=\mu(0)$. Then $x * y, y * x \in S$. Since $S$ is an either-or set over $(X, *, 0)$, either $\{x, y\} \subseteq S$ or $\{x, y\} \subseteq S^{c}$. It follows that either $\mu(x)=$ $\mu(y)=1$ or $\mu(x)=\mu(y)=0$. Hence $\mu$ is a fuzzy- $d$-subset of $(X, *, 0)$. 


\section{Conflict of Interests}

The authors declare that there is no conflict of interests regarding the publication of this paper.

\section{Acknowledgment}

The authors are grateful to the referee for valuable suggestions and help.

\section{References}

[1] K. Iséki and S. Tanaka, "An introduction to theory of BCKalgebras," Mathematica Japonica, vol. 23, pp. 1-26, 1978.

[2] K. Iséki, “On BCI-algebras," Mathematics Seminar Notes, vol. 8, pp. 125-130, 1980.

[3] J. Neggers and H. S. Kim, "On $d$-algebras," Mathematica Slovaca, vol. 49, pp. 19-26, 1999.

[4] J. S. Han, H. S. Kim, and J. Neggers, "Strong and ordinary $d$ algebras," Journal of Multiple-Valued Logic and Soft Computing, vol. 16, no. 3-5, pp. 331-339, 2010.

[5] H. S. Kim, J. Neggers, and K. S. So, "Some aspects of $d$-units in d/BCK-algebras," Journal of Applied Mathematics, vol. 2012, Article ID 141684, 10 pages, 2012.

[6] H. S. Kim and J. Neggers, "The semigroups of binary systems and some perspectives," Bulletin of the Korean Mathematical Society, vol. 45, no. 4, pp. 651-661, 2008.

[7] H. F. Fayoumi, "Locally-zero groupoids and the center of $\operatorname{Bin}(X)$," Communications of the Korean Mathematical Society, vol. 26, no. 2, pp. 163-168, 2011.

[8] J. S. Han, H. S. Kim, and J. Neggers, "The hypergroupoid semigroups as generalizations of the groupoid semigroups," Journal of Applied Mathematics, vol. 2012, Article ID 717698, 8 pages, 2012. 


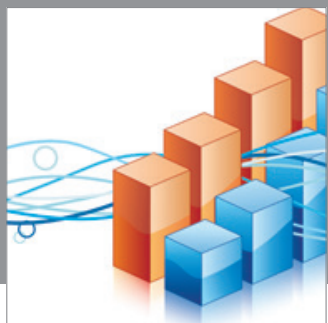

Advances in

Operations Research

mansans

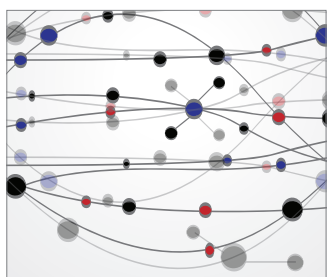

The Scientific World Journal
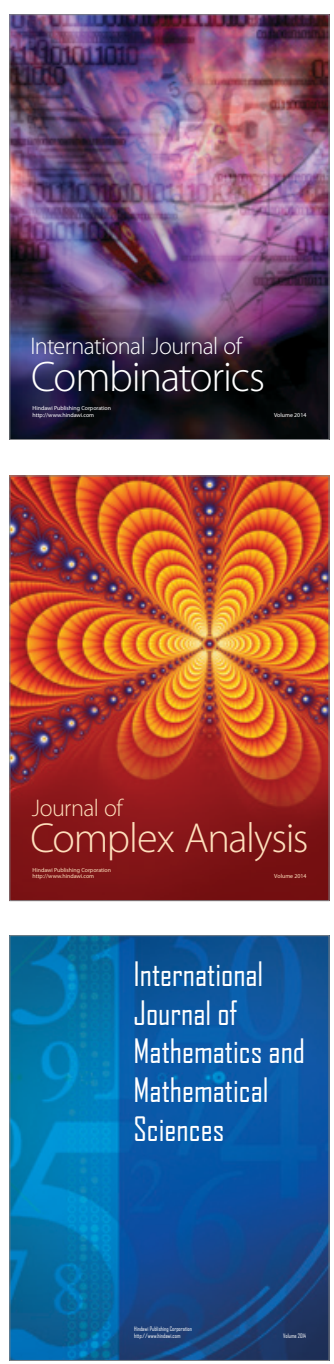
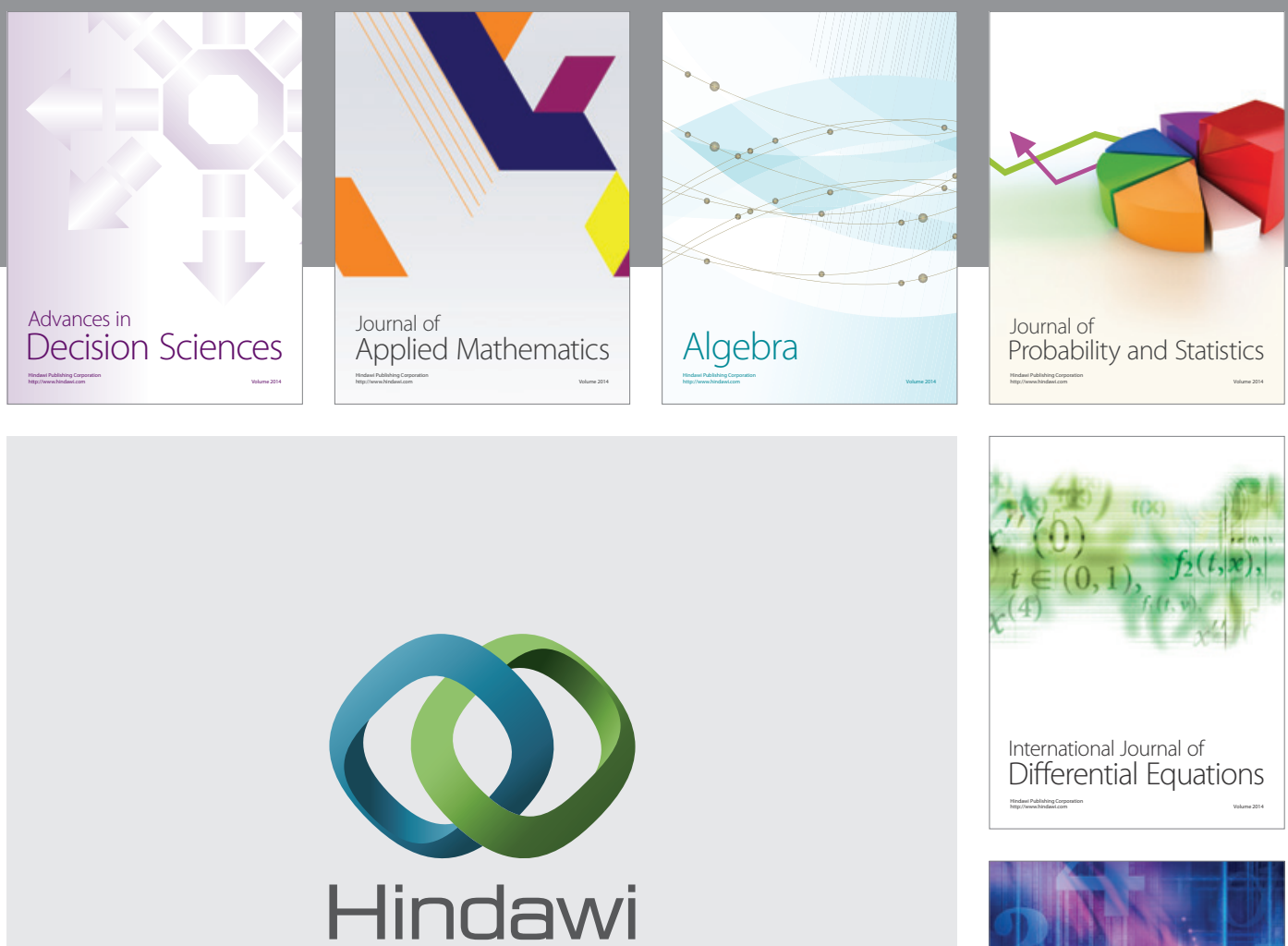

Submit your manuscripts at http://www.hindawi.com
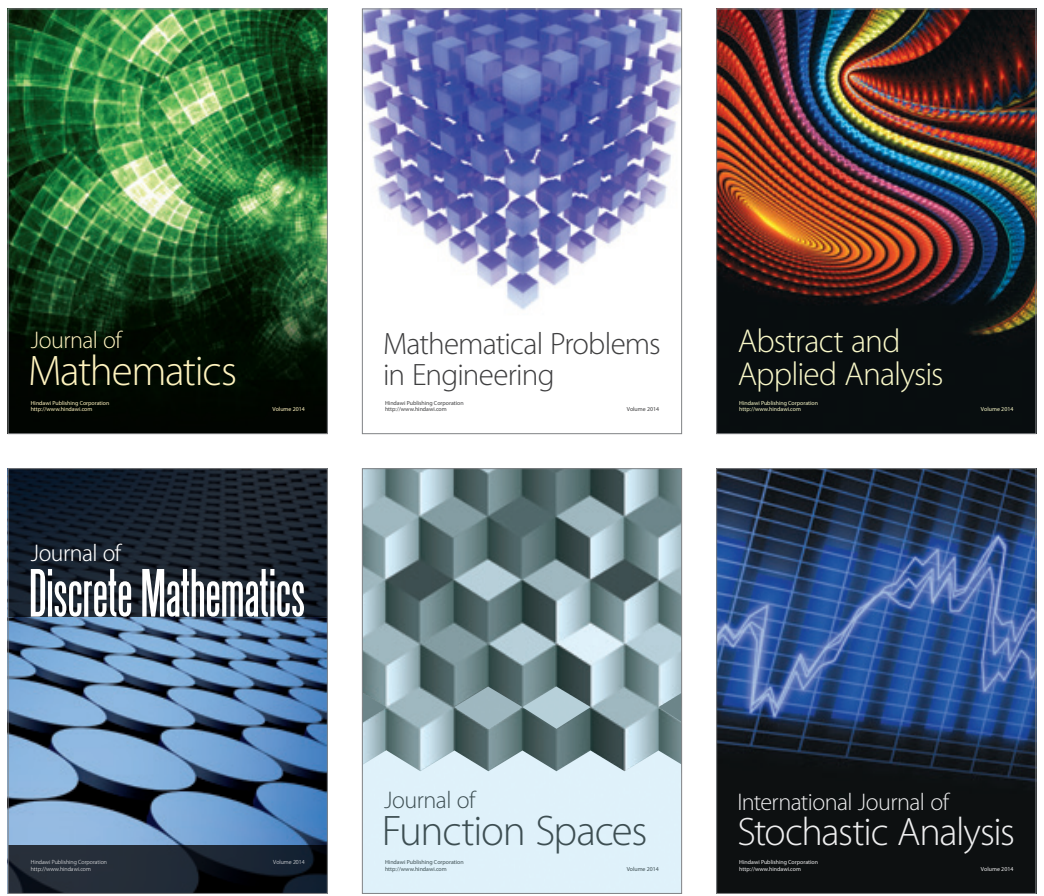

Journal of

Function Spaces

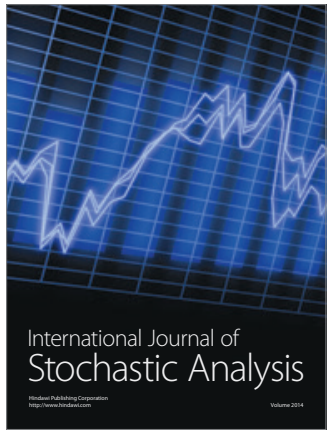

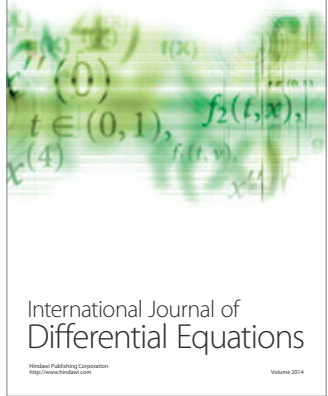
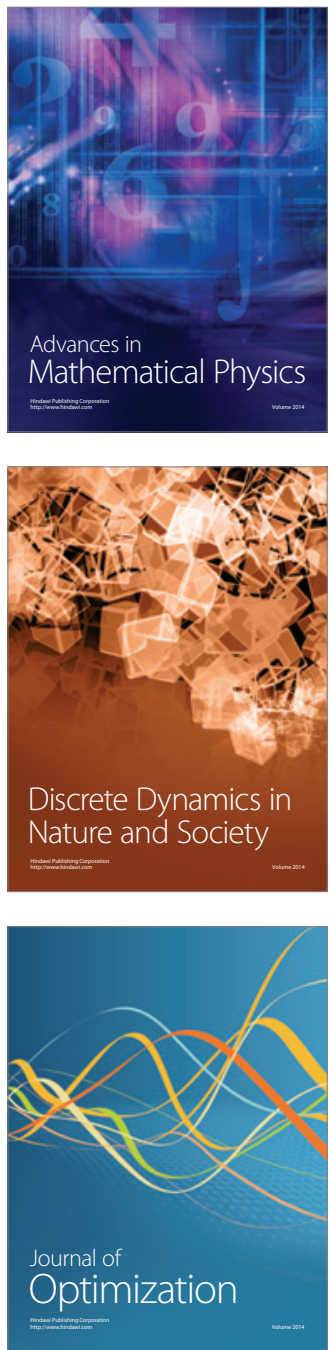